\title{
Comparative Analysis of Different Deployment Techniques in Wireless Sensor Network
}

\author{
${ }^{1}$ Thrimurthi M, ${ }^{2}$ Adithya B A, ${ }^{3}$ Thandavamurthi H R \\ ${ }^{1,2,3}$ Department of Electronics and Communication \\ Malnad College of Engineering \\ Hassan, India.
}

\begin{abstract}
In this communication era, wireless sensor network places a vital role. Wireless sensor network comprises of various types of sensor networks. Deployment of sensor nodes in wireless sensor network is a major concern to optimal result. There are various techniques in deploying the wireless sensor node. Among those some of the methods are, randomized method of deployment, grid based deployment, contour based deployment and projection based deployment. In this paper we are comparing all above methods and we show that projection based method out performs the rest of all othermethods.
\end{abstract}

Keywords- Coverage; Connectivity; Deployment; Energy

\section{INTRODUCTION}

Wireless sensor network is a collection of various wireless sensor nodes for sensing and transcieving operation. The basic unit of wireless sensor network is wireless sensor node. The wireless sensor node comprises power unit, processor, transceiver, analog to digital converter and sensors as shown in Figure 1.1. The process of arranging wireless sensor nodes in a wireless sensor network in appropriate manner is called sensor deployment [14].

The message transmitting and receiving by a wireless sensor node in a wireless sensor network consumes energy, which is a major factor for determining the performance of sensor network. Wireless sensor network batteries are exhaustible. Less complexity and a better manageability is also one of the major concerns in deployment of wireless sensor node.

\author{
${ }^{4}$ Gururaj H L \\ Department of Computer science and Engineering \\ Malnad College of Engineering \\ Hassan, India.
}

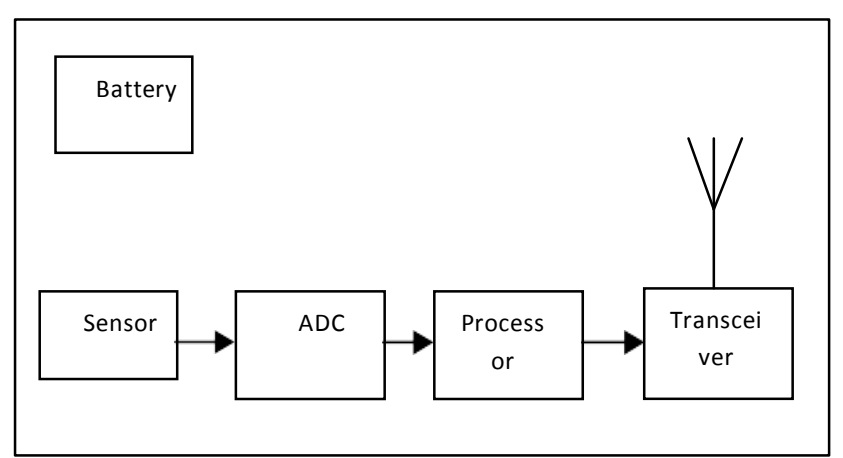

Figure 1.1 Sensor Node

Deploying the sensors nodes is a facet of conceptual energy utilization in wireless sensor network. It is necessary to optimize energy consumption in various ways. The range of communication of different sensor nodes depends on its own application. We have various deployment methods such as random deployment method, grid deployment method, contour based deployment method and projection based deployment method.

Wireless sensor networks work with two methods, inter cluster and intracluster. Wireless sensor network having various applications namely weather forecasting, military applications, law enforcement and security application, location based identification, environmental sensing and monitoring.

The paper is organized as follows section two explains the literature survey, section three explains the comparative results on various deployment methods and at last in section four draws the conclusion of our research work.

\section{RELATEDWORK}

In wireless sensor network deployment of wireless nodes is major concern to ensure the entire region of target area with minimum sensor nodes. The Figure 2.1 shows the random deployment of sensor nodes. The conventional method of deploying the sensor nodes in a sensor network is randomized. In general, a uniform random deployment is assumed to be 
easy as well as cost effective. In this methodology the nodes are placed in such a way that nodes are deployed randomly in a target area in irrespective of the range of communication [1] [6]. The major pitfall of this technique is that the range of communication of sensor node will overlap with the range of communication of other sensor node. In this method due to its randomness we cannot say that the exact number of sensors present in a particular region. Here by which the energy consumed by the sensor node is comparatively high. As the nodes are deployed randomly this may or may not cover the complete target area, due to its randomness [13].

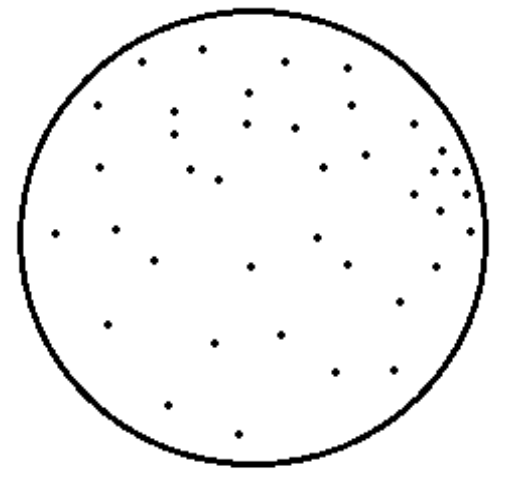

Figure 2.1 Random deployments of sensor nodes in a given area

An efficient method of deployment has been proposed to overcome the drawback of random deployment called grid based deployment [7]. In this method the target area is completely divided into horizontal rows and vertical columns. Such intersection of a row and a column is called grid. This methodology is also called as precise methodology.

In this method the deployment of sensor node grid depends on the grid size and the range of the communication of sensor nodes. Here the numbers of sensors are fixed for 1 square [13]. So it is easy to calculate total number of sensors present in the network. Here the maximum utilization of sensing in each grid results to the efficient performance of the sensor network is comparatively done. The major applications of sensor network are weather forecasting, detecting extent animals at the forest, in battlefield and in agriculture field [9]. Where we may not expect the target area to be in a square shape as depicted in Figure 2.2. Hence this methodology is also called as square methodology [2]. Grid methodology cannot be applied for irregular shape of the area [8]. Also the main drawback of grid scan is that the grid size is hard to decide. Too large a size will result in poor accuracy and too small a size will result high computation cost due to large number of grids.
Volume: 02, Issue: 04, April 2017

ISBN: 978-0-9957075-5-9

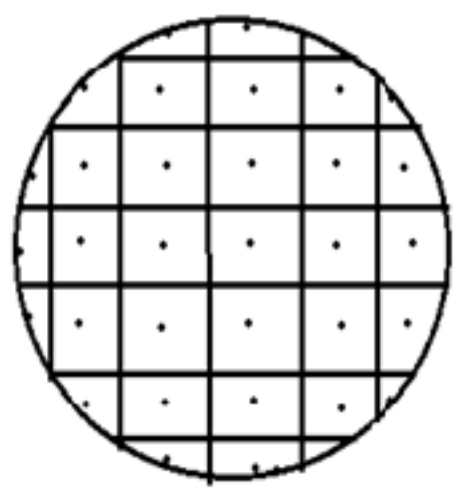

Figure 2.2 Grid based sensor node deployment technique.

A new approach has been developed to overcome the drawback of grid methodology called contour methodology. In this methodology the sensor nodes are arranged sequentially without any overlapping range of communication [4]. This deployment method can be applied to any shape of the (irregular) target area [6]. The sensor nodes are placed in a circular shape. Therefore, it founds difficult to place the sensor nodes at the border of the target area. The disadvantage of this methodology is if we try to deploy the sensor nodes on the border, an uncovered area will be created which is called as a hole, by which extra sensors required to cover the holes it leads to overlapping of sensor nodes' range of communication as shown in Figure 2.3. It also requires maximum number of nodes but actually not required in that border. Hence this deployment method consumes more energy and also expensive [3][10].

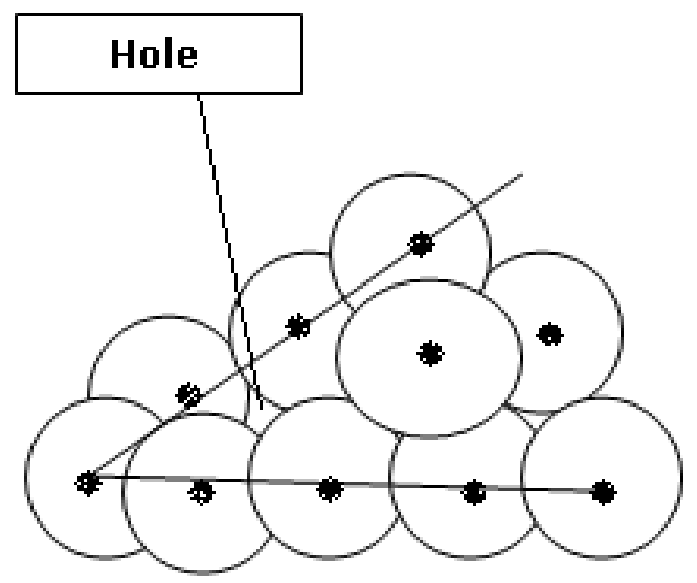

Figure 2.3 Coverage hole that is undetected by Contour based method

An efficient method of deployment has been proposed to overcome the drawback of previous methodologies called projection based methodology [4]. This method shows an improved performance over the contour based method. In this technique the covering of entire plane which do not overlap nor leave any gaps. It allows full coverage of area [5]. So we get good quality of information, and there is no chance of loss of information. This method can be applied for any irregular shapes to ensure the full target area with minimum number of sensor nodes. The range of communication of sensor node is not fixed [12]. In this methodology the sensor nodes are 
arranged in a hexagonal shape as depicted in the below Figure2.4. Deployment at the border in projection based and is shown in the below Figure 2.5. The range of communication without overlapping of every node is and node to node distance is $r \sqrt{ } 3$ where ' $r$ ' is the radius of the range of communication [4] [8].

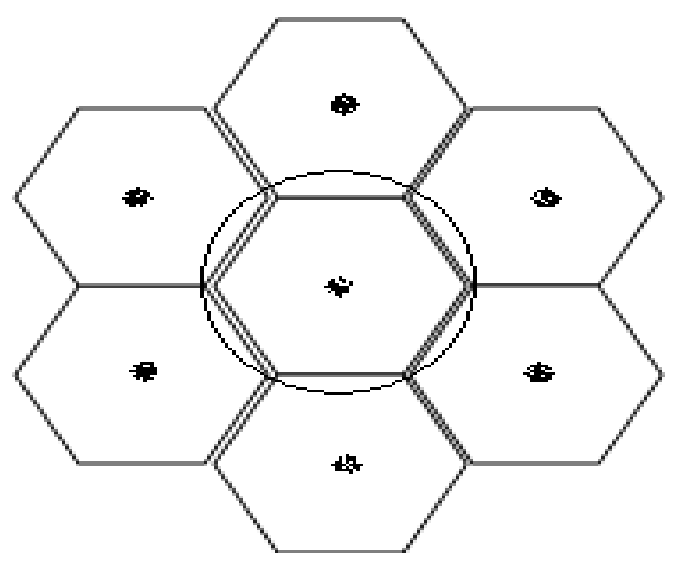

Figure 2.4 Hexagon Deployment of sensor nodes

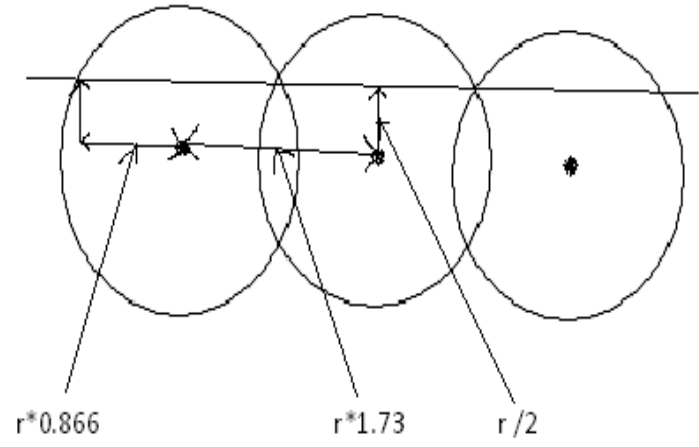

Figure 2.5 Deployment at the border in projection based

\section{COMPARATIVE Resultanalysis}

In this section we conduct a performance evaluation of 4 deployment strategies. The primary factors for all experiments are: The number of nodes, sensing range and energy consumption. We had simulated all the deployment models as we had discussed in the above section using an event driven simulator NS-2(Network Simulator-2). We had compared and analyzed the four different deployment methods considering the energy utilization, number of required sensor nodes for coverage and connectivity of sensor network [5] [11].

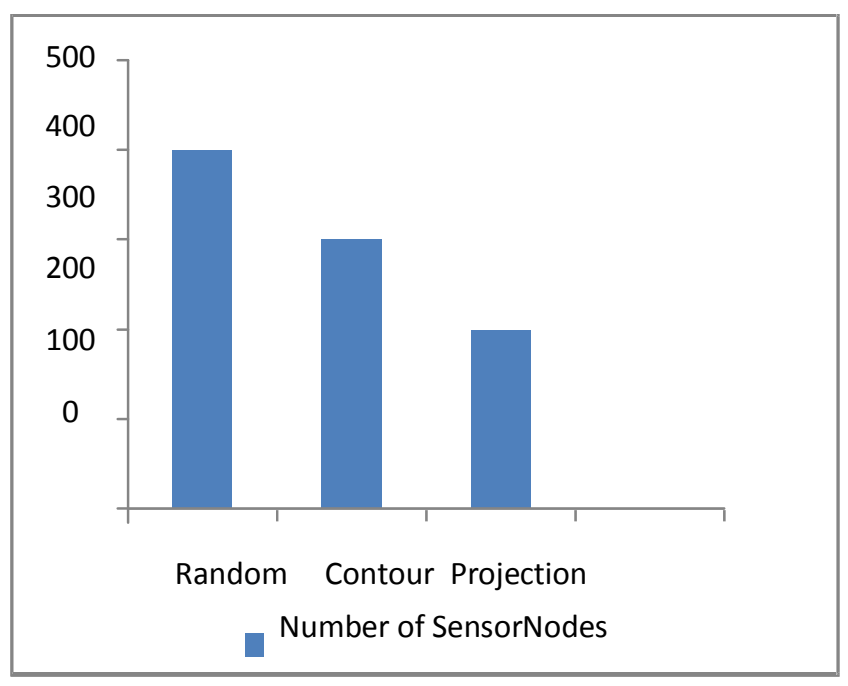

Figure 3.1 Number of sensor nodes in Regular Area

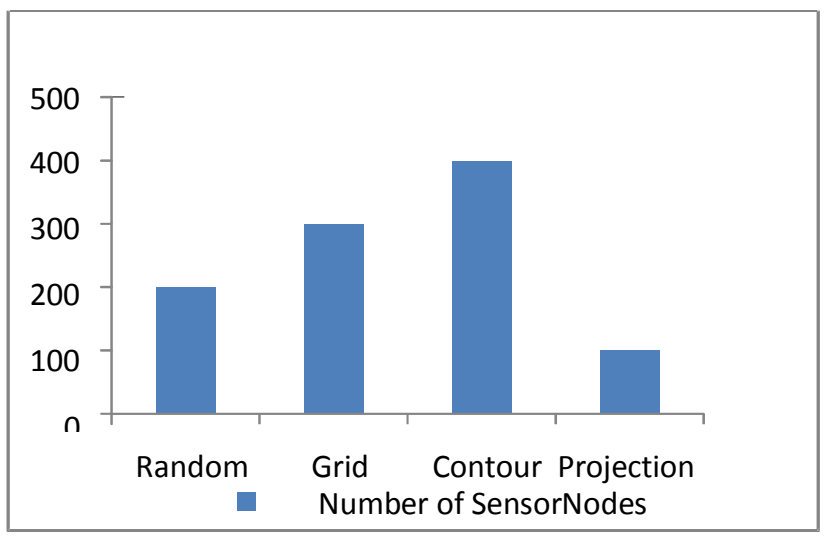

Figure 3.2 Number of sensor nodes in Irregular area

In the above Figure 3.1 the random deployment and contour method utilizes the maximum number of sensor nodes compare to grid deployment and projection based method in regular shape. As grid topology cannot be applied for irregular shape. We compared rest of the three deployment methods for irregular shape in the below depicted Figure 3.2.

\section{A. Energy Utilization}

The consumption of energy is major concern in Wireless sensor network. A sensor node is composed of sensing unit, a processing unit, a transceiver unit and a power unit. The main consumer of energy is transceiver unit and processing unit. The processing unit requires energy to aggregate data, computer routing and maintain security etc.... As projection based method consumes less energy compared to other deployment methods. The energy utilization graph depicts as below Figure3.3.

By this comparative analysis the projection based methodology gives an efficient result compared to other three 
discussed methods. And it is also not expensive. Result projection based method outperforms differently.

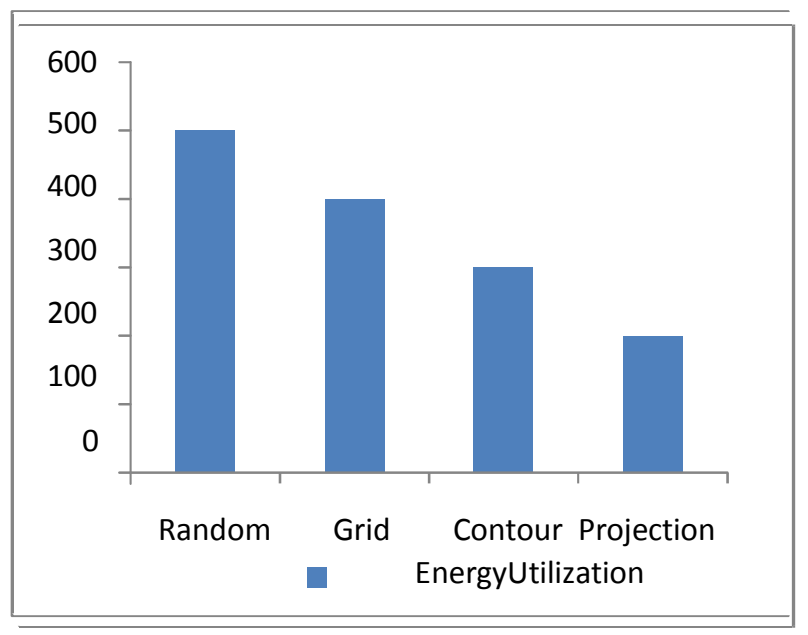

Figure 3.3 Energy Utilization

\section{B. Memory Usage}

The consumption of memory is major concern in wireless sensor network, sensor node contains several units. It used to receive a data surrounded by sensor node. The projection based method is requiring less memory as compared to other deployment for full coverage and connectivity of target area.

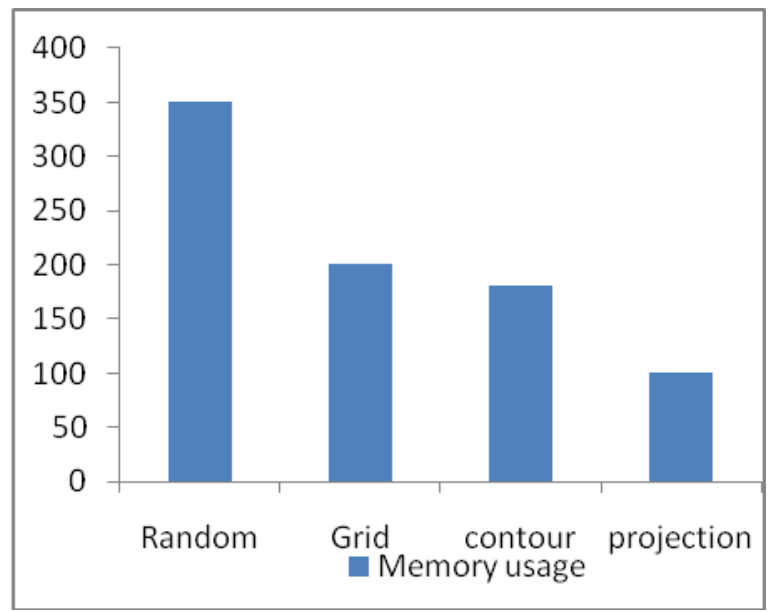

Figure 3.4 Memory Usage

In this above Figure 3.4 shows the projection based method is consuming less memory to store data. Hence this method gives efficient result compare to other three discussed methods.

\section{CONCLUSION}

With the major progress in technology miniaturization and increase in reduction of cost many deploying methods are supported in Wireless coverage and connectivity energy utilization. As a result projection based method outperforms the other deployment methods. This method is not so expensive to build and gives optimum results. The sensing and message transmission is also done very efficiently in projection based methods. In our further research work, we intended to work on various network parameters for comparing the deployment techniques by considering with and without obstacles and also aim to develop and implement a new more efficient universally acceptable deployment method for wireless sensor network. Deployment methods. This method is not so expensive to build and gives optimum results. The sensing and message transmission is also done very efficiently in projection based methods. In our further research work, we intended to work on various network parameters for comparing the deployment techniques by considering with and without obstacles and also aim to develop and implement a new more efficient universally acceptable deployment method for wireless sensor network.

\section{REFERENCES}

[1] M. Li, D. R. Ding, and T. L. Guo, "Random deployment Strategy of Wireless Sensor Network Node", Computer Engineering, Vol. 38, No. 5, 2012, pp.99-101

[2] Manijeh Keshtgari1, AmeneDeljoo "A Wireless Sensor Network Solution for Precision Agriculture Based on Zig-Bee Technology Wireless Sensor Network",http://dx.doi.org//0.4236/wsn.2012.41002,publishedonli ne,January2012/http://www.scirp.org/journal/wsn.

[3] Haisheng Tan, Yuexuan Wang, XiaohongHao, Qiang-Sheng Hua, and Francis C.M. Lau "Arbitrary Obstacles Constrained Full Coverage in Wireless Sensor Networks" Pandurangan et al. (Eds.): WASA 2010, LNCS 6221, pp. 1-10, 2010.c_ SpringerVerlag Berlin Heidelberg 2010

[4] I. Khoufi, P. minet, A. Laouiti“ A simple method for the deployment of Wireless sensors to ensure full coverage of an irregular area with obstacles" ACM, 2014

[5] X Bai, S. kumar, D. Xuan "Deploying Wireless sensors to achieve both coverage and connectivity" MobiHoc'06,May 22-05-2006, Florence, Itlay .copyright 2006, ACM.1-59593-368-9/06/0005.

[6] Haitao Zhang and Cuiping Liu A "Review on Node Deployment of Wireless Sensor Network", IJCSI International Journal of Computer Science Issues,Vol.9,Issue6,No3,November2012ISSN(Online): 16940814www.IJCSI.org.

[7] X. He, X. L. Gui, and J. An, “A Deterministic Deployment Approach of Nodes in Wireless Sensor Networks for Target Coverage", Journal of XiAnJiaotong University Vol. 44,No.6, 2010, pp.6-10.

[8] S. Dhillon, and K. Chakrabarty, "Sensor Placement for Effective coverage and surveillance in distributed sensor networks", in Proceedings of the IEEE Wireless Communications and Networking Conference, 2003, Vol. 3, pp.1609-1614 .

[9] K. Konstantinos, X. Apostolos, K. Panagiotis and S. George, "Topology Optimization in Wireless Sensor Networks for Precision Agriculture Applications," Sensor Communication, 2007, pp. 526-530.

[10] Guey-Yun Chang, Shan-Hung Wu, Yen- Ting Chen, Jang-Ping Sheu. "Adaptive k-Coverage Contour Evaluation and Deployment in Wireless Sensor Networks". ACM transactions on Sensor Networks, vol.9, no.4, article 40, publication date: July 2013

[11] PallaviSahu, Sunil R. Gupta. "Deployment technique in wireless sensor network", International journal of soft computing and engineering[IJSCE], ISSN:2231-2307, volume-2, Issue-3, July 2012. 
[12] Sabu M Thampi, NithyaAniyan, Chitharanjan, "Hexagonal Groups Based Key Management Using Deployment Knowledge in Wireless Sensor Networks", International conference on advance computing communications and informatics(ICACCI-2012)

[13] Wintyipoe, Jens B.Schmitt, "Node deployment in long wireless sensor Networks: Coverage, energy consumption and worst-case delay". AINTEC '09 November 18-20-2009, Bankok,Thailand.copy right 2009 ACM 978-1-60558-614-4/09/11.

[14] Ramesh B., Gururaj H. L., and Chandrika J. "Network Performance Comparative Analysis of Torus and Modified Mesh Interconnections with Source Routing for Packet Loss", International Journal of Computer Theory and Engineering, Vol. 7, No. 4, August 2015.

\section{AUTHORS PROFILE}

Thrimurthi $\mathbf{M}$ pursuing Electronics and Commucication Engineering in Malnad Cllege of Engineering, Hassan. He is a Student member of IEEE Computer Society, Bengalore Section . His research iterests are Communication System, Embedded System, Multimedia Networking.

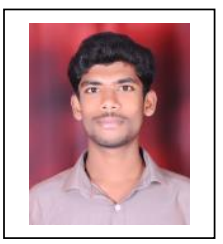

Thandavamurthi $\mathbf{H} \mathbf{R}$, pursuing Electronics and Commucication Engineering in Malnad Cllege of Engineering, Hassan. He is a Student member of IEEE Computer Society, Bengalore Section . His research iterests are Communication System, Embedded System, Multimedia Networking.

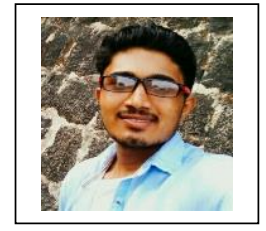

Gururaj H L, received B.E (2010) and M.Tech (2013) in Computer Science and Engineering from Visvesvaraya Technological University, Belagaum , Karnataka. $\mathrm{He}$ is pursuing his doctoral degree in Malnad College of Engineering, Hassan. Currently he is working as an Assistant Professor in the Department of Computer Science

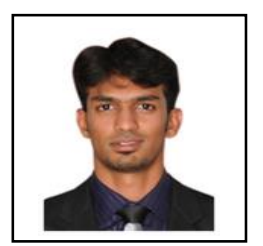
and Engineering, Malnad College of Engineering, Hassan, India. He is a member of IEEE Computer Society, Bangalore Section. He has awarded with Young Scientist International Travel Support under SERB, DST, Govt of India in December 2016. He has research interests in congestion control algorithms, security issues in cloud computing and routing protocols for multihop wireless networks, QoS-aware routing algorithms in ad hoc networks and multimedia networks.
Adithya B A, pursuing Electronics and Commucication Engineering in Malnad Cllege of Engineering, Hassan. $\mathrm{He}$ is a Student member of IEEE Computer Society, Bengalore Section. His research iterests are Communication System, Embedded System, Multimedia Networking

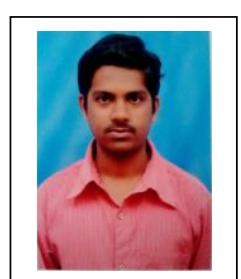

(C) 2017 by the author(s); licensee Empirical Research Press Ltd. United Kingdom. This is an open access article distributed under the terms and conditions of the Creative Commons by Attribution (CC-BY) license. (http://creativecommons.org/licenses/by/4.0/). 